$\mathrm{E}$

EVALUAR
2018, Vol. 18, No 1.

ISSN $1667-4545$

Recuperado de https://revistas.unc.edu.ar/index.php/revaluar

Laboratorio de Evaluación Psicológica y Educativa

Facultad de Psicología - Universidad Nacional de Córdoba

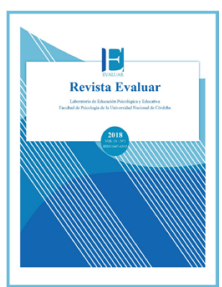

\title{
Propiedades psicométricas del inventario de ideación suicida positiva y negativa PANSI
}

\section{Psychometric properties of the Positive and Negative Suicide Ideation Inventory (PANSI)}

\author{
Bertha Avendaño-Prieto ${ }^{1}$, Mónica Pérez-Prada ${ }^{2}$, Mildred Vianchá-Pinzón ${ }^{2}$, \\ Lizeth Martínez-Baquero ${ }^{2}$, Ronald Toro ${ }^{1}{ }^{*}$ \\ 1 - Universidad Católica de Colombia. Bogotá DC., Colombia. \\ 2 - Universidad de Boyacá. Tunja, Boyacá, Colombia.
}

Introducción

Método

Resultados

Discusión

Referencias

Recibido: 20/09/2017 Revisado: 30/10/2017 Aceptado: 07/12/2017

\section{Resumen}

La conducta suicida como problemática de salud mental requiere de un manejo con herramientas precisas de evaluación, particularmente en la ideación suicida. Se llevó a cabo un estudio instrumental para establecer las propiedades psicométricas del Inventario de Ideación Suicida Positiva y Negativa (PANSI) en una muestra de 1318 estudiantes voluntarios entre 13 y 19 años, que cursaban entre octavo y undécimo grado en tres ciudades colombianas. Los análisis factoriales exploratorio y confirmatorio mostraron la estructura teórica esperada y se obtuvo un ajuste favorable de los ítems según el modelo de Rasch (excepto el ítem 4). Además, los resultados de consistencia interna fueron $\alpha=.77$ para la Ideación Suicida Positiva y $\alpha=.89$ para la Ideación Suicida Negativa. Nuestros datos indican que el PANSI es un instrumento con evidencias de constructo y consistencia interna para el objetivo y contexto para el que fue elaborado, por lo que proponemos un PANSI final de 13 ítems útil en población adolescente capaz de identificar las dos dimensiones de la ideación suicida.

Palabras clave: comportamiento suicida, ideación suicida, salud mental, análisis factorial confirmatorio, Teoría de Respuesta al Ítem (TRI), adolescentes

\begin{abstract}
Suicide behavior as a mental health problem requires a good management with improved assessment tools, particularly for suicide ideation. An instrumental study was carried out to establish the psychometric properties of the Positive and Negative Suicide Ideation Inventory (PANSI) in 1318 students, 13 to 19 years-old, from three cities in Colombia, with ages from, and from attending 8th to 11th secondary school levelsgrade in three cities in Colombia. The expected theoretical structure was obtained by exploratory and confirmatory factorial analysis. Also, a favorable fit was found, according to the Rasch model (except item 4). Moreover, when internal consistencies were analyzed, a value of $\alpha=.77$ was obtained for positive suicide ideation, while $\alpha=.89$ was shown for negative suicide ideation. These data indicate that PANSI presents construct validity and consistency, according to the purpose and context in which it was elaborated. Therefore, we suggest the use of a 13 items PANSI in high school students able to identify the two dimensions of suicidal ideation.
\end{abstract}

Keywords: suicidal behavior, suicide ideation, mental health, confirmatory factor analysis, Item Response Theory (IRT), adolescents

Cómo citar este artículo: Avendaño-Prieto, B., Pérez-Prada, M., Vianchá-Pinzón, M., Martínez-Vaquero, L., \& Toro, R. (2018). Propiedades psicométricas del inventario de ideación suicida positiva y negativa PANSI. Revista Evaluar, 18(1), 27-39. Recuperado de https://revistas.unc.edu.ar/index. php/revaluar

*Correspondencia a: Ronald Toro, Universidad Católica de Colombia, Facultad de Psicología, Avenida Caracas \# 46-22, Casa de Psicología, bloque K, Bogotá, Colombia. Teléfono (+571) 3277300. ratoro@ucatolica.edu.co 


\section{Introducción}

La conducta suicida, definida como toda acción producida con el propósito explícito o implícito de quitarse la vida, incluye cualquier intención, ideación o pensamiento que pueda culminar en la muerte (Toro-Tobar, Avendaño-Prieto, \& Castrillón, 2016). Durante la historia de la humanidad esta conducta ha sido parte de rituales de purificación, expiación o de seguimiento a las pautas socio culturales propias de las diferentes épocas (Andrade-Salazar, 2012).

Sin embargo, en la última década las cifras de suicidio han aumentado de manera tal que instituciones como la Organización Panamericana de la Salud y la Organización Mundial de la Salud (OPS, OMS, 2016), lo consideran un problema de salud pública que ha sido incluido para su prevención en el Plan Estratégico (2014 a 2019) como uno de los indicadores de impacto a evaluar. $\mathrm{Si}$ bien dicho informe indica que el número de muertes por suicidio representa el dato más visible y dramático, sería importante considerar también otras modalidades de conducta suicida que incluyen la ideación, la planificación y el intento de suicidio.

La OMS define la ideación suicida (IS), como pensamientos pasivos sobre querer estar muerto o pensamientos activos sobre quitarse la vida uno mismo, no necesariamente acompañados de conductas de preparación para lograrlo (OMS, 2012). La IS hace referencia no solo a su carácter persistente, fijo u obsesivo sino también a la comunicación de la ideación, ya sea de manera verbal o escrita. Cuando una persona habla de querer morirse o desear suicidarse, estos llamados de atención deben ser considerados seriamente por los profesionales de la salud mental, sobre todo si son reiterativos (Aja, 2007).

Desde una perspectiva cognitivo conductual, cualquier persona que se plantea el suicidio experimenta pensamientos o ideas suicidas. Para poder prevenir cualquier tipo de conducta suicida es de gran importancia en el ámbito terapéutico que el profesional sea capaz de reconocer signos de inconformidad en el paciente, así como signos de insatisfacción con su modo de vivir, y así detectar de forma temprana la presencia de ideación suicida (Martí, 2013).

Colombia no está exenta de este flagelo; en el 2015 se registró un aumento del 10\% en el número de suicidios con relación al año anterior, de los cuales el $80 \%$ de las víctimas eran varones. El grupo etario entre 15 y 34 años fue la población más afectada. La proporción de lesiones fatales autoinfligidas fue de 5.22 eventos por cada 100 mil habitantes mayores de cinco años, aproximadamente un $28 \%$ más que la proporción promedio de los últimos nueve años (4.08 eventos por cada 100 mil habitantes). Sobre los métodos utilizados para llevar a cabo el suicidio, el ahorcamiento fue el mecanismo más común en varones, seguido de intoxicación o envenenamiento, siendo este último el cual más frecuente en mujeres (Instituto Nacional de Medicina Legal y Ciencias Forenses, 2017).

Ante la magnitud de la problemática, se han desarrollado instrumentos de evaluación que permiten identificar la ideación suicida y cuyo objetivo es determinar hasta qué punto las ideas suicidas pueden llegar a materializarse mediante la muerte por suicidio. Uno de los instrumentos de evaluación de IS más conocidos es la Escala de Ideación Suicida o SSI (del inglés Scale for Suicide Ideation; Beck, Kovacs, \& Weissman, 1979), diseñada para evaluar las actitudes, las conductas y los planes de cometer actos suicidas. Otro instrumento es el cuestionario de ideación suicida o Suicidal Ideation Questionnaire (SIQ; Reynolds, 1987) que consiste en 25 ítems para evaluar la frecuencia e intensidad de los pensamientos, y también comportamientos suicidas. Este cuestionario 
se basa en una escala de 0 a 6 , que incluye desde "nunca lo he pensado" hasta "todos los días", con un total de 150 puntos y un solo factor. El SIQ puede ser aplicado de forma grupal, es útil para evaluar programas de intervención y prevención debido a su sensibilidad y capacidad discriminatoria de la detección del riesgo suicida, y presenta una elevada consistencia interna $(\alpha=.97)$, una medida de comparación test-retest favorable $(r=$ .86), y correlaciones significativas con depresión, desesperanza, ansiedad y autoestima (valores de $r_{s}$ entre .38 y .60).

Uno de los instrumentos más utilizados es el Inventario de Ideación Suicida Positiva y Negativa (PANSI; Osman, Gutiérrez, Kopper, Barrios, \& Chiros, 1998), que permite medir los factores tanto de protección como de riesgo de ideación suicida. Los autores de PANSI realizaron dos estudios: el primero se administró una versión de 20 ítems a 150 estudiantes de sexo masculino y 300 estudiantes de sexo femenino, y se realizó un análisis factorial con rotación varimax. Se conservaron dos factores: la ideación positiva y la ideación negativa. En el segundo estudio se realizó un análisis factorial confirmatorio a fin de validar el ajuste de los modelos de un factor, y un análisis oblicuo de dos factores en otra muestra de 84 hombres y 202 mujeres. El modelo oblicuo de dos factores proporcionó un excelente ajuste. Además, los autores examinaron la evidencia preliminar de validez concurrente y predictiva, revisando su correlación con las medidas de los síntomas depresivos $(\beta=.49, t=5.89, p<.001)$, y desesperanza $(\beta=.16, t=2.10, p<.001)$.

Posteriormente, Osman et al. (2003) evaluaron la estructura factorial del PANSI, utilizando el análisis factorial confirmatorio (AFC). Los 195 participantes eran pacientes psiquiátricos hospitalizados, entre 14 y 17 años de edad. De acuerdo al AFC, el mejor ajuste fue el del modelo oblicuo de 2 factores. Los autores también revelaron que las estimaciones de confiabilidad de consistencia interna de las dos subescalas de PANSI, PANSINegativo y PANSIPositivo, fueron elevadas $(\alpha=.96$ y $\alpha=.89$, respectivamente); estas puntuaciones en las escalas PANSI presentaron diferencias significativas entre aquellos que intentaron suicidarse y los controles, y aquellos en riesgo severo de suicidio y controles, con un $90 \%$ de sensitividad y un $92.6 \%$ de especificidad. Los análisis correlacionales proporcionaron un fuerte respaldo para la validez concurrente de las escalas. Los análisis de regresión logística respaldaron el uso de este nuevo inventario como medida de riesgo de los comportamientos relacionados con el suicidio, ya que los puntajes en las escalas PANSI $(n=54)$ mostraron evidencia satisfactoria en relación con la confiabilidad test-retest durante un período de 2 semanas.

La IS positiva (ISP) contempla una serie de pensamientos protectores contra el suicidio; por ejemplo: “Has sentido que tenías el control de la mayoría de las situaciones de tu vida?” o "¿Te sentiste alegre porque te estaba yendo bien en el colegio o en el trabajo?"; mientras que la IS negativa (ISN) contempla ítems de riesgo suicida, como "¿Pensaste en matarte porque no tenías esperanza en el futuro?" o "¿Pensaste que tus problemas eran tan graves que la única opción que tenías era suicidarte?". El PANSI ha obtenido un modelo bifactorial de la IS con una puntuación por cada factor y una medida total de IS (Osman et al., 2003).

El instrumento PANSI fue adaptado a poblaciones de Colombia por Villalobos-Galvis (2009). En este estudio, la adaptación se realizó en 643 participantes con escolaridad de sexto grado de bachillerato hasta el quinto año de universidad. Mediante un análisis factorial exploratorio se obtuvieron los dos factores originales del instrumento: uno positivo (pensamientos protectores contra el suicidio) y otro negativo (ideas suicidas) con 
$64 \%$ de varianza total. Esta adaptación demostró poseer una estructura bifactorial como la que obtuviera previamente el instrumento en una población inglesa (Osman et al., 1998), un apropiado índice de confiablidad (alfa de Cronbach $\alpha=.90$ para la escala completa; $\alpha=.93$ para ideas negativas, y $\alpha=.84$ para ideas positivas) y adecuadas asociaciones $(p<.01)$ con las medidas de desesperanza, depresión, ideación suicida y autoestima que evidenciaron la validez concurrente. Villalobos-Galvis (2009) concluyó que el PANSI tiene buenas propiedades psicométricas, razón por la cual podría ser aplicado en estudiantes adolescentes y jóvenes.

El PANSI se ha utilizado en diferentes estudios en población colombiana. Por ejemplo, Quiceno y Vinaccia (2013) lo emplearon para evaluar las relaciones entre la orientación positiva hacia el futuro, la felicidad y la IS como indicadores de la calidad de vida, en 291 adolescentes de colegios públicos de la ciudad de Bogotá. Los resultados fueron: una asociación positiva y moderada entre las IS positivas (primera escala del PANSI) en la dimensión de calidad de vida y bienestar psicológico $(r=.41, p<.001)$, y una correlación negativa pero débil entre las ideas negativas (segunda escala del PANSI) en la dimensión relación padres/vida familiar de calidad de vida infantil $(r$ $=-.363, p<.001)$.

En otro estudio que aplicó PANSI, llevado a cabo por Siabato-Macias y Salamanca-Camargo (2015) en estudiantes universitarios, se encontró que aproximadamente el $30 \%$ de los evaluados presentaba un nivel alto de ISN. A su vez, Forero, Siabato y Salamanca (2017) en un estudio con adolescentes, encontraron que el 28\% presentaba un nivel alto de ISN. En cuanto a la asociación IS y trastornos de la conducta alimentaria, por medio del PANSI y de otros tests, otro estudio mostró que un $18 \%$ de los adolescentes evaluados piensa en el suicidio como una alternativa para solucio- nar sus conflictos, y que un $42 \%$ de los participantes con síntomas de anorexia y bulimia presentaron alta ISN (Martínez-Baquero, Vianchá-Pinzón, Pérez-Prada, \& Avendaño-Prieto, 2017).

Este instrumento ha sido utilizado como medida de convergencia para el desarrollo de nuevos instrumentos; por ejemplo, el diseño de Toro-Tobar et al. (2016) de un inventario llamado IDIS (Inventario de Desesperanza e Ideación Suicida), que permite evaluar cognición suicida con base en los modelos teóricos de la desesperanza y la IS y en el que los valores que arroja el PANSI son evidencia de validez convergente. En este estudio se obtuvo una correlación favorable con el puntaje total de IS $(r=.68 ; p<.001)$, considerado favorable para el nuevo instrumento.

Teniendo en cuenta tanto la aplicabilidad del PANSI como la carencia de análisis de poblaciones colombianas por medio de la Teoría de Respuesta al Ítem (TRI) y de AFC, analizamos las propiedades psicométricas del instrumento PANSI en población adolescente. Los objetivos específicos fueron: establecer evidencia de la validez de constructo de PANSI mediante un AFC, observar la estabilidad de los ítems en una estructura de dos factores, analizar el ajuste de los ítems respecto de la TRI y establecer la confiabilidad de la prueba a través del coeficiente alfa de Cronbach y el coeficiente Omega.

\section{Método}

Tipo de estudio

Se utilizó una metodología de tipo instrumental (Ato, López, \& Benavente, 2013). Se analizaron las características psicométricas del PANSI siguiendo la Teoría Clásica de los Tests (TCT) y la TRI. Dentro de la TCT, se realizó el AFE, el AFC y la confiabilidad con los coeficientes alfa de Cronbach y Omega. A partir de la TRI, 
se estableció el ajuste de los ítems al modelo de Rasch.

\section{Participantes}

Los participantes fueron seleccionados mediante muestreo no probabilístico por conveniencia (Hernández-Sampieri, Fernández-Collado, \& Baptista-Lucio, 2010). Se solicitó la participación de los colegios de las poblaciones objeto de estudio, pero no todos aceptaron la invitación. El estudio se realizó en 1318 estudiantes, 57.2\% varones y $42.8 \%$ mujeres entre 13 y 19 años de edad $(\mathrm{M}=15.43, \mathrm{DE}=1.33)$, estudiantes de octavo, noveno, décimo y undécimo grado en tres ciudades colombianas. Como criterios de inclusión se consideraron la voluntad de participar en el estudio y la firma del consentimiento informado.

\section{Instrumentos}

El instrumento utilizado fue el Inventario de Ideación Suicida Positiva y Negativa (PANSI), validado por Villalobos-Galvis (2009) en una población de estudiantes de Colegios y Universidades de San Juan de Pasto del departamento de Nariño, Colombia.

Este instrumento presenta una lista de catorce ítems que se refieren a pensamientos suicidas presentes en las últimas dos semanas anteriores a la aplicación de la escala. Ocho de los catorce ítems evalúan ideación suicida negativa, equivalente a factores de riesgo, y los otros seis evalúan ideación suicida positiva, que corresponde a factores protectores, los que en su totalidad permiten determinar la frecuencia de aparición de cada uno de los catorce pensamientos. Los participantes deben responder haciendo uso de una escala tipo Likert (de 5 puntos) con niveles entre 0 (nunca) y
4 (siempre). Las preguntas número 2, 6, 8, 12, 13 y 14 corresponden a ideación positiva; mientras que las preguntas número 1, 3, 4, 5, 7, 9, 10 y 11 corresponden a la ideación negativa.

\section{Procedimiento}

Se seleccionaron dos instituciones educativas por ciudad, de las cuales se tomó al azar un curso por grado. Posteriormente, los padres de familia recibieron el consentimiento informado. Antes de la aplicación del instrumento se les expresó con claridad a los participantes el objetivo del estudio y la forma en que se utilizaría la información obtenida; asimismo, se les explicó el diligenciamiento del instrumento mediante instrucciones estandarizadas y se le solicitó a cada participante el consentimiento informado firmado. El estudio se realizó de acuerdo con la legislación vigente promulgada en la resolución número 8430 del año 1993 (Ministerio de Salud, 1993), en la que se dictan las normas científicas, técnicas y administrativas para la investigación en salud, y también en la práctica de investigaciones en psicología de acuerdo con la Ley No 1090 (2006), mediante la cual se establece el Código Deontológico y Bioético del ejercicio de la psicología en Colombia. Se les aseguró también a los estudiantes que su participación no involucraba ningún riesgo para su integridad física o mental, se les garantizó la confidencialidad y se les indicó que su participación era voluntaria.

Los resultados de la Teoría Clásica de los Tests se analizaron con el software SPSS versión 22 (IBM Corp., 2013) y los de la Teoría de Respuesta al Ítem, con Winsteps 3.69.1.13 (Linacre, 2016). Posteriormente se llevaron a cabo los análisis estadísticos y psicométricos. Para determinar la validez de constructo, se dividió aleatoriamente la muestra en dos grupos; el grupo conforma- 
do por 671 estudiantes esta se analizó mediante análisis factorial exploratorio y posteriormente, mediante TRI (específicamente con el modelo de Rasch para ítems politómicos) se estableció el ajuste de los ítems. Después de eliminar el ítem que no se ajustó al modelo, se repitió el AFE. Los resultados obtenidos en este análisis sirvieron de base para el AFC de la segunda muestra, conformada por 647 estudiantes. Finalmente, se determinó la consistencia interna del instrumento mediante el coeficiente Omega de McDonald.

\section{Resultados}

Evidencia de validez de constructo

Para procesar los datos obtenidos en la muestra de 671 participantes, se determinó el índice de adecuación muestral de Kaiser-Meyer-Olkin (KMO), cuyo valor fue .871. Mediante el índice de esfericidad de Bartlett, se encontraron valores de $\chi^{2}=4187.589$ y $p<.01$, los que permitieron realizar el análisis factorial exploratorio. Se encontraron tres factores, el primero explicó el $37.77 \%$ de la varianza, el segundo el $16.07 \%$ y el tercero el 7.51, de modo que resultaba explicado un total de $61.36 \%$ de la varianza. Se utilizó rotación oblimin, asumiendo que los dos factores evalúan el mismo constructo aunque de forma inversa y siguiendo el criterio utilizado tanto en la prueba original como en la adaptación (Tabla 1).

Según la distribución de los ítems, el Factor 1 representa la ideación negativa, el Factor 2 quedó conformado por cuatro de los seis ítems $(2,6,8$ y 12$)$ de ideación positiva y el Factor 3 , compuesto de los ítems 13 y 14 de ideación positiva, conforma un solo factor. No se observaron cargas cruzadas. En el análisis realizado por Villalobos-Galvis con este instrumento (2009) también se utilizó la rotación oblicua, y a diferencia de los resultados observados en este estudio, se encontraron dos factores no independientes, dado que los ítems 13 y 14 que constituyen la ideación suicida positiva compartían una carga factorial en dos factores, aunque la mayor carga se encontraba en el factor esperado.

Posteriormente, en los ítems de cada factor se evaluaron los parámetros de ajuste cercano y lejano en los ítems de cada factor mediante la Teoría de Respuesta al Ítem, de acuerdo al modelo de Rash. La Tabla 2 muestra los resultados obtenidos para ISN.

Encontramos que el ítem 4 no se ajustó al modelo, ya que sus valores de INFIT y OUTFIT (1.96 y 1.99, respectivamente) no están incluidos en el intervalo aceptado [.4; 1.40$]$, es decir que la calibración con respecto al supuesto de unidimensionalidad no se cumple para este ítem y por lo tanto es una medida anómala con respecto al rasgo latente (Attorresi, Lozzia, Abal, Galibert, \& Aguerri, 2009; Bond \& Fox, 2007; Muñiz, 2010).

A diferencia de lo observado en el factor ISN, en el factor de ideación suicida positiva, todos los ítems se ajustaron al modelo (Tabla 3). Por ello, se volvió a realizar el AFE con la exclusión del ítem 4. Encontramos que el índice KMO $=.877 \mathrm{y}$ el índice de esfericidad de Bartlett resultó en $\chi^{2}=3945.740$ y $p<.01$. En este análisis se encontraron dos factores: el primer factor correspondió al 39.09\% de la varianza, mientras que el segundo correspondió al 17.21\%, lo que totalizó $56.31 \%$. Los ítems 13 y 14 estuvieron incluidos en el factor al que pertenecen (Ideación Suicida Positiva), como muestra la Tabla 4.

Una vez establecida la validez de constructo de la prueba y observada la estabilidad de los ítems en una estructura de dos factores, se sometió este modelo a un AFC. Para ello, se utilizó el módulo AMOS de SPSS, versión 22. Los índices de bondad de ajuste obtenidos fueron los siguientes: la proporción entre $\chi^{2}$ y el número de grados de libertad correspondientes $\left(\chi^{2} / g l\right)$, cuyo 
Tabla 1

Distribución de ítems según AFE del PANSI.

\section{Ítems}

1. ¿Has considerado seriamente matarte porque no pudiste cumplir con lo que otras personas esperaban de ti?

2. ¿Has sentido que tenías el control de la mayoría de las situaciones de tu vida?

3. ¿Pensaste en matarte porque no tenías esperanza en el futuro?

4. ¿Te sentiste tan triste por tu relación con alguien importante, que quisiste estar muerto?

5. ¿Pensaste en matarte porque no pudiste hacer algo que era muy importante en tu vida?

6. ¿Tuviste esperanza en el futuro porque las cosas estaban saliendo como tu querías?

7. ¿Pensaste en matarte porque no encontraste una solución a un problema personal?

8. ¿Te sentiste alegre porque te estaba yendo bien en el colegio o en el trabajo?

9. ¿Pensaste en matarte porque viste que tu vida era un fracaso?

10. ¿Pensaste que tus problemas eran tan graves que la única opción que tenías era suicidarte?

11. ¿Te sentiste tan solo(a) o tan triste que querías matarte para así terminar con ese sufrimiento?

12. ¿Tuviste confianza en las capacidades que tenías para enfrentar la mayoría de problemas de tu vida?

13. ¿Sentiste que valía la pena vivir la vida?

14. ¿Tuviste confianza en lograr tus metas en el futuro?

F1 (ítems F2 (4 ítems F3 (2 ítems

de ISN) ISP) de ISP)

.63

$-.31$

\begin{tabular}{|c|c|c|}
\hline .23 & .58 &, 00 \\
\hline .78 & .10 & -.13 \\
\hline .62 & -.23 & .4 \\
\hline .78 & -.02 & .02 \\
\hline-.04 & .78 & -.03 \\
\hline .77 & -.04 & -.01 \\
\hline-.12 & .62 & .07 \\
\hline .85 & .00 & .00 \\
\hline .77 & .07 & -.2 \\
\hline .84 & -.01 & .04 \\
\hline-.04 & .63 & .33 \\
\hline-.17 & .18 & .75 \\
\hline-.12 & .31 & .71 \\
\hline
\end{tabular}

Nota. F (Factor), ISN (Ideación Suicida Negativa), ISP (Ideación Suicida Positiva).

Tabla 2

Ajuste del modelo de Rasch para los ítems de Ideación Suicida Negativa.

\begin{tabular}{|c|c|c|c|c|}
\hline Item & Medida & Error & INFIT & OUTFIT \\
\hline $\begin{array}{l}\text { 4. ¿Te sentiste tan triste por tu relación con alguien importante, que } \\
\text { quisiste estar muerto? }\end{array}$ & -.55 & .07 & 1.96 & 1.99 \\
\hline $\begin{array}{l}\text { 1. ¿Has considerado seriamente matarte porque no pudiste cumplir } \\
\text { con lo que otras personas esperaban de ti? }\end{array}$ & .47 & .08 & 1.13 & 1.20 \\
\hline $\begin{array}{l}\text { 7. ¿Pensaste en matarte porque no encontraste una solución a un } \\
\text { problema personal? }\end{array}$ & -.24 & .07 & 1.00 & 1.03 \\
\hline $\begin{array}{l}\text { 5. ¿Pensaste en matarte porque no pudiste hacer algo que era muy } \\
\text { importante en tu vida? }\end{array}$ & .03 & .08 & .94 & .97 \\
\hline $\begin{array}{l}\text { 11. ¿Te sentiste tan solo(a) o tan triste que querías matarte para así } \\
\text { terminar con ese sufrimiento? }\end{array}$ & -.36 & .07 & .80 & .82 \\
\hline $\begin{array}{l}\text { 10. ¿¡Pensaste que tus problemas eran tan graves que la única opción } \\
\text { que tenías era suicidarte? }\end{array}$ & .18 & .08 & .79 & .73 \\
\hline 3. ¿Pensaste en matarte porque no tenías esperanza en el futuro? & .44 & .08 & .79 & .68 \\
\hline 9. ¿Pensaste en matarte porque viste que tu vida era un fracaso? & .04 & .07 & .66 & .65 \\
\hline
\end{tabular}

Nota. Estadístico estandarizado de ajuste cercano (INFIT), y estadístico estandarizado de ajuste lejano (OUTFIT). 
Tabla 3

Ajuste del modelo de Rasch para los ítems del factor de Ideación Suicida Positiva.

\begin{tabular}{|c|c|c|c|c|}
\hline Ítem & Medida & Error & INFIT & OUTFIT \\
\hline $\begin{array}{l}\text { 2. ¿Has sentido que tenías el control de la mayoría de las situaciones de } \\
\text { tu vida? }\end{array}$ & .96 & .05 & 1.41 & 1.42 \\
\hline $\begin{array}{l}\text { 8. ¿Te sentiste alegre porque te estaba yendo bien en el colegio o en el } \\
\text { trabajo? }\end{array}$ & -.25 & .05 & 1.07 & 1.03 \\
\hline $\begin{array}{l}\text { 6. ¿Tuviste esperanza en el futuro porque las cosas estaban saliendo } \\
\text { como tu querías? }\end{array}$ & .42 & .04 & .98 & .97 \\
\hline 13. ¿Sentiste que valía la pena vivir la vida? & -.06 & .05 & .95 & .86 \\
\hline $\begin{array}{l}\text { 12. ¿Tuviste confianza en las capacidades que tenías para enfrentar la } \\
\text { mayoría de problemas de tu vida? }\end{array}$ & .09 & .04 & .81 & .79 \\
\hline 14. ¿Tuviste confianza en lograr tus metas en el futuro? & -.59 & .05 & .80 & .68 \\
\hline
\end{tabular}

Nota. INFIT (estadístico estandarizado de ajuste cercano), OUTFIT (estadístico estandarizado de ajuste lejano).

Tabla 4

Distribución de ítems por factor, con exclusión del ítem 4

Ítems

F1 (ítems

de ISN, sin

el ítem 4)

1. ¿Has considerado seriamente matarte porque no pudiste cumplir con lo que otras personas esperaban de ti?

2. ¿Has sentido que tenías el control de la mayoría de las situaciones de tu vida?

3. ¿Pensaste en matarte porque no tenías esperanza en el futuro?

5. ¿Pensaste en matarte porque no pudiste hacer algo que era muy importante en tu vida?

6. ¿Tuviste esperanza en el futuro porque las cosas estaban saliendo como tu querías?

7. ¿Pensaste en matarte porque no encontraste una solución a un problema personal?

8. ¿Te sentiste alegre porque te estaba yendo bien en el colegio o en el trabajo?

9. ¿Pensaste en matarte porque viste que tu vida era un fracaso?

10. ¿Pensaste que tus problemas eran tan graves que la única opción que tenías era suicidarte?

11. ¿Te sentiste tan solo(a) o tan triste que querías matarte para así terminar con ese sufrimiento?

12. ¿Tuviste confianza en las capacidades que tenías para enfrentar la mayoría de problemas de tu vida?

13. ¿Sentiste que valía la pena vivir la vida?

14. ¿Tuviste confianza en lograr tus metas en el futuro?
.73

.29

.82

.76

F2 (ítems

de ISP)

.05

.76

$-.05$

$-.06$

.61

.83

$-.01$

.83

$-.03$

.82

.00

$-.03$

.76

$-.31$

.57

$-.24$

.67

Nota. F(Factor), ISN(Ideación suicida negativa), ISP(Ideación suicida positiva). 
valor fue 3.585; el índice global de ajuste (GFI), que resultó en .95; y el índice de ajuste comparativo (CFI), con un valor igual a .96. Los valores de estos índices oscilan entre 0 y 1 ; cero indica ausencia de ajuste, mientras que 1 indica ajuste óptimo; valores de .95 o superiores son considerados excelentes (Pilatti, Godoy, \& Brussino, 2012). El CFI compara el ajuste del modelo en cuestión a un modelo nulo, en el que todos los ítems son independientes (Kline, 2000). El valor del error cuadrado de aproximación a las raíces medias (RMSEA) fue de .06. Un valor de RMSEA igual o menor que .05 es considerado un índice óptimo, $\mathrm{y}$ es considerado aceptable en el rango $[.05 ; .08]$ (Hu \& Bentler, 1999). Así, en nuestro estudio, el intervalo de confianza del RMSEA fue aceptable. La Figura 1 muestra el diagrama de la estructura resultante, con las covarianzas entre los errores de medida elaboradas con base en los índices de modificación.

\section{Análisis de Consistencia Interna y Confiabilidad}

Mediante el coeficiente alfa de Cronbach se determinó la consistencia interna de cada uno de los factores: el factor Ideación Suicida Positiva obtuvo un valor de $\alpha=.77 \mathrm{y}$ el factor Ideación Suicida Negativa obtuvo un valor de $\alpha=.89$. Valores cercanos a 1 reflejan una mayor evidencia de la confiabilidad del instrumento; mientras que valores de alfa entre .5 y .79 son considerados pobres o débiles (como el de la escala ISP), y valores de alfa entre .8 y .9 se consideran buenos como el de la escala ISN- (Carvajal, Centeno, Watson, Martínez, \& Sanz-Rubiales, 2011). Finalmente, se computó la confiabilidad Omega (McDonald, 1970), índice que indica consistencia interna de una escala, y se obtuvo un valor igual a .81 para el factor Ideación Suicida Positiva y un valor igual a .93 para Ideación Suicida Negativa.

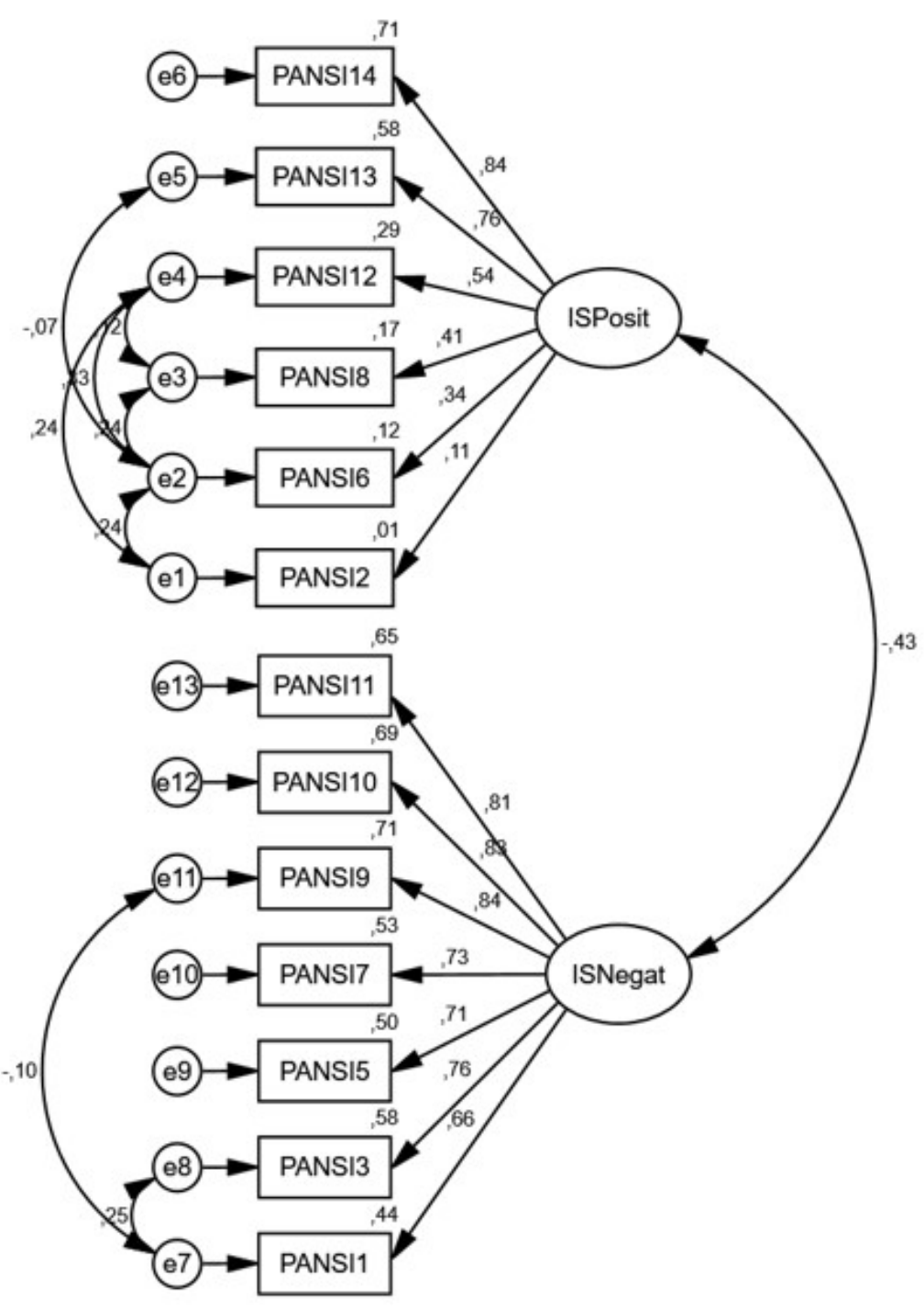

Figura 1

Gráfico del análisis factorial confirmatorio (AFC) con las estimaciones estandarizadas.

\section{Discusión}

El objetivo de nuestro estudio fue llevar a cabo una revisión de las propiedades psicométricas del PANSI como parte del mejoramiento continuo de los instrumentos disponibles para la detección y prevención del riesgo suicida en población adolescente. Para ello, se efectuó una revisión de la estructura del test a nivel factorial y un análisis de confiabilidad, teniendo en cuenta las consideraciones teóricas y metodológicas del 
inventario original y la validación para la población colombiana.

Con respecto a la estructura de la escala utilizada, encontramos que el ítem 4 no se ajustó al modelo según la TRI, posiblemente debido a su estructura semántica asociada a síntomas depresivos, duelo o afrontamiento emocional en particular. Al excluir el ítem 4 se evidenció la estructura bifactorial original del instrumento; es decir, se mantuvieron las dos escalas propuestas por Osman et al. (1998) y Villalobos-Galvis (2009). Acorde a los indicadores de medición OUTFIT e INFIT, el resto de los ítems que constituyen la escala se mantuvieron dentro de los parámetros recomendados (Muñiz, 2010; Attorresi et al., 2009).

A diferencia del estudio original y la revisión posterior (Osman et al., 1998; Osman et al., 2003, respectivamente) o la adaptación de Villalobos-Galvis (2009), la consistencia interna del factor ideación suicida negativa fue de $\alpha=.89 \mathrm{y}$ el alfa del factor ideación suicida positiva de $\alpha$ $=.77$, alfas considerados bueno y débil respectivamente (Carvajal et al., 2011), mientras que las anteriores revisiones obtuvieron coeficientes superiores a los encontrados en la presente investigación, incluida la reciente adaptación en Malasia, en la que se lograron coeficientes de confiabilidad alfa entre .93 y .84 (Sinniah et al., 2015). Ante estas discrepancias, se puede afirmar que los alfas obtenidos en este estudio son aceptables como indicadores de confiabilidad del PANSI. Por lo tanto, podemos afirmar que esta nueva versión del PANSI cuenta con propiedades psicométricas adecuadas para su uso en estudiantes escolarizados colombianos de octavo a undécimo grados.

\section{Limitaciones y estudios futuros}

Dado que el suicidio es una de las principales causas de muerte prematura prevenible mediante intervenciones oportunas, basadas en datos fidedignos y a menudo de bajo costo (OMS, 2014), los esfuerzos destinados a explorar su diagnosis resultan relevantes para cualquier población humana. Por este motivo, se propone, por lo que se propone continuar realizando estudios con el PANSI que permitan identificar la ideación suicida en otras poblaciones, sobre todo considerando que no se tuvieron en cuenta las diferencias étnicas en la muestra conformada para el presente estudio.

Una de las principales limitaciones del estudio fue el no haber incluido indicadores de nivel de riesgo, ya que la conducta suicida se suele presentar en tres momentos específicos: ideación, intento y muerte (Li, Bao, Li, \& Wang, 2016). En el caso del instrumento PANSI, se recomienda estudiarlo en términos de capacidad discriminativa de los niveles de riesgo, dado que una persona con intento de suicidio suele presentar la IS desde mucho antes de ser diagnosticada.

Otra limitación importante fue la falta de formulación de una pregunta control que sirviera como criterio de riesgo externo al instrumento.

Además, no pudimos determinar los límites de sensibilidad y especificidad del instrumento, ya que no realizamos un contraste entre muestra clínica y no clínica. Por esta misma razón tampoco pudimos establecer un punto de corte que indicara a partir de qué puntuación se presentaría el rasgo evaluado.

En futuras revisiones psicométricas de este instrumento por otros autores, el ítem eliminado (ítem 4 del PANSI) debería ser incluido, a fin de poder observar la invarianza con respecto al modelo resultante del presente estudio.

En conclusión, podemos afirmar que el PANSI presenta propiedades psicométricas favorables que lo convierten en una medida apropiada de la ideación suicida, tanto positiva como negativa. Este instrumento tiene validez y confiabilidad 
adecuadas, y debido a su corta extensión y a su facilidad de calificación por parte de los profesionales de salud mental, su empleo es recomendable como escala de evaluación de la conducta suicida en adolescentes escolarizados capaces de brindar autoinforme.

\section{Referencias}

Aja, L. (2007). El suicidio y los factores indicadores de riesgo. Primer Congreso Latinoamericano de Educación, Bogotá, Colombia.

Andrade-Salazar, J. A. (2012). Aspectos psicosociales del comportamiento suicida en adolescentes. Revista electrónica de Psicología Iztacala, 15(2), 688-721. Recuperado de http://www.revistas.unam.mx/index. $\mathrm{php} / \mathrm{repi} /$ index

Ato, M., López, J. J., \& Benavente, A. (2013). Un sistema de clasificación de los diseños de investigación en psicología. Anales de Psicología, 29(3), 1038-1059. doi: 10.6018/analesps.29.3.178511

Attorresi, H. F., Lozzia, G. S., Abal, F. J., Galibert, M. S., \& Aguerri, M. E. (2009). Teoría de Respuesta al Ítem. Conceptos básicos y aplicaciones para la medición de constructos psicológicos. Revista Argentina de Clínica Psicológica, 18(2), 179-188. Recuperado de http:/www.clinicapsicologica.org.ar/

Beck, A. T., Kovacs, M., \& Weissman, A. (1979). Assessment of suicidal intention: The Scale for Suicide Ideation. Journal of Consulting and Clinical Psychology, 47(2), 343-352. doi: 10.1037/0022-006X.47.2.343

Bond, T. G., \& Fox, C. M. (2007). Applying the Rash Model: Fundamental measurement in the human sciences ( $2^{\mathrm{a}}$ ed.). New Jersey, USA: Lawrence Erlbaum Associates Publishers.

Carvajal, A., Centeno, C., Watson, R., Martínez, M., \& Sanz-Rubiales, A. (2011). ¿Cómo validar un instrumento de medida de la salud? Anales del Sistema Sanitario de Navarra, 34(1), 63-72. doi: 10.4321/ s1137-66272011000100007
Forero, I., Siabato, E., \& Salamanca, Y. (2017). Ideación suicida, funcionalidad familiar y consumo de alcohol en adolescentes de Colombia. Revista Latinoamericana de Ciencias Sociales, Niñez y Juventud, 15(1), 431-442. Recuperado de http://revistaumanizales. cinde.org.co/

Hernández-Sampieri, R., Fernández-Collado, C., \& Baptista-Lucio, M. (2010). Metodología de la investigación. México: Mc Graw Hill.

Hu, L. T., \& Bentler, P. M. (1999). Cutoff criteria for fit indices in covariance structure analysis: Conventional criteria versus new alternatives. Structural Equation Modeling A Multidisciplinary Journal, 6(1), 1-55. doi: 10.1080/10705519909540118

IBM Corp. (2013). IBM SPSS Statistics for Windows, Version 22.0. [software de cómputo]. Armonk, NY: IBM Corp.

Instituto Nacional de Medicina Legal y Ciencias Forenses (2017). Forensis 2016. Datos para la vida, 18(1). Recuperado de http://www.medicinalegal.gov.co/ web/guest/inicio

Kline, P. (2000). Handbook of Psychological Testing. Londres, Inglaterra: Routledge.

Ley $\mathrm{N}^{\circ}$ 1090. (2006). Por la cual se reglamenta el ejercicio de la profesión de Psicología, se dicta el Código Deontológico y Bioético y otras disposiciones. Colombia. Recuperado de http://www.secretariasenado. gov.co/senado/basedoc/ley_1090_2006.html

Li, D., Bao, Z., Li, X., \& Wang, Y. (2016). Perceived school climate and Chinese adolescents' suicidal ideation and suicide attempts: The mediating role of sleep quality. Journal of School Health, 86(2), 75-83. doi: 10.1111/josh.12354

Linacre, J. M. (2016). A User's Guide to WINSTEPS-MINISTEP Rasch-Model Computer Programs Program Manual. Recuperado de http://www.winsteps.com/ winman/copyright.htm

Martí, M. (2013). Protocolo para la detección y manejo inicial de la ideación suicida. Guía desarrollada por el Centro de Psicología Aplicada (CPA). Universidad Autónoma de Madrid (UAM). Recuperado de 
https:/www.uam.es/centros/psicologia/paginas/cpa/ paginas/doc/documentacion/rincon/protocolo_ideacion_suicida.pdf

Martínez-Baquero, L. C., Vianchá-Pinzón, M. A., PérezPrada, M. P., \& Avendaño-Prieto, B. L. (2017). Asociación entre conducta suicida y síntomas de anorexia y bulimia nerviosa en escolares de Boyacá, Colombia. Acta Colombiana de Psicología, 20(2), 178-188.

McDonald, R. P. (1970). Theoretical foundations of principal factor analysis and alpha factor analysis. British Journal of Mathematics and Statistical Psychology, 23(1), 1-21. doi: 10.1111/j.2044-8317.1970. tb00432.x

Ministerio de Salud (1993). Resolución 8430 de 1993. Por el cual se establecen las normas cientificas, técnicas y administrativas para la investigación en salud. Disponible en https://www.minsalud.gov.co/sites/ rid/Lists/BibliotecaDigital/RIDE/DE/DIJ/RESOLUCION-8430-DE-1993.PDF

Muñiz, J. (2010). Las teorías de los tests: Teoría clásica y teoría de respuesta a los ítems. Papeles del Psicólogo, 31(1), 57-66. Recuperado de http://www.papelesdelpsicologo.es/pdf/1796.pdf

Organización Mundial de la Salud (2012). For which strategies of suicide prevention is there evidence of effectiveness? Copenhagen, Denmark: WHO Regional Office for Europe. Recuperado de http://www.euro. who.int/en/home

Organización Mundial de la Salud (2004). El suicidio, un problema de salud pública enorme y sin embargo prevenible, según la OMS. (Informe del 8 de Septiembre). Recuperado de www.who.int/entity/ mediacentre/news/releases/2004/pr61/es/

Organización Mundial de la Salud (2014). Prevención del suicidio, un imperativo global. Ginebra, OMS. Recuperado de http://www.who.int/mental_health/ suicide-prevention/es/

Organización Panamericana de la Salud (2016). Prevención de la conducta suicida. Washington, DC: Autor. Recuperado de http://iris.paho.org/xmlui/bitstream/ handle/123456789/31167/9789275319192-spa.pdf

Osman, A., Gutiérrez, P. M., Kopper, B. A., Barrios, F. X., \& Chiros, C. E. (1998). The positive and negative suicide ideation inventory: Development and validation. Psychological Reports, 82(3), 783-793. doi: 10.2466/pr0.1998.82.3.783

Osman, A., Gutiérrez, P. M., Kopper, B. A., Barrios, F. X., Linden, S. C., \& Truelove, R. S. (2003). A preliminary validation of the Positive and Negative Suicide Ideation (PANSI) Inventory with normal adolescent samples. Journal of Clinical Psychology, 59(4), 493512. doi: 10.1002/jclp.10154

Pilatti, A., Godoy, J. C., \& Brussino, S. (2012). Adaptación de instrumentos entre culturas: Ejemplos de procedimientos seguidos para medir las expectativas hacia el alcohol en el ámbito argentino. Trastornos Adictivos, 74(2), 58-64. doi: 10.1016/S1575-0973(12)70045-4

Quiceno, J. M., \& Vinaccia, S. (2013). Calidad de vida, factores salutogénicos e ideación suicida en adolescentes. Terapia Psicológica, 31(2), 263-271. doi: 10.4067/S0718-48082013000200012

Reynolds, W. M. (1987). Suicidal Ideation Questionnaire: Professional Manual. USA, Florida: Psychological Assessment Resources.

Siabato-Macias, E. F., \& Salamanca-Camargo, Y. (2015). Factores asociados a ideación suicida en universitarios. Psychologia, 9(1), 71-81. doi: 10.21500/19002386.994

Sinniah, A., Oei, T. P. S., Chinna, K., Shah, S. A., Maniam, T., \& Subramaniam, P. (2015). Psychometric properties and validation of the Positive and Negative Suicide Ideation (PANSI) inventory in an outpatient clinical population in Malaysia. Frontiers in Psychology, 21(6), 19-34. doi: 10.3389/fpsyg.2015.01934

Toro-Tobar, R. A., Avendaño-Prieto, B. L., \& Castrillón, D. A. (2016). Design and psychometric analysis of the Hopelessness and Suicide Ideation Inventory "IDIS". International Journal of Psychological Research, 9(1), 52-63. doi: 10.21500/20112084.2100

Villalobos-Galvis, F. H. (2010). Validez y fiabilidad del inventario de ideación suicida positiva y negativa 
- PANSI, en estudiantes colombianos. Universitas Psychologica, 9(2), 509-520. Recuperado de http:// revistas.javeriana.edu.co/index.php/revPsycho/ index/ 